\title{
BMJ Open Research protocol: a realist synthesis of cross-border patient mobility from low-income and middle-income countries
}

\author{
Jo Durham, Sarah J Blondell
}

To cite: Durham J, Blondell SJ. Research protocol: a realist synthesis of cross-border patient mobility from low-income and middle-income countries. BMJ Open 2014;4:e006514. doi:10.1136/bmjopen-2014006514

- Prepublication history and additional material is available. To view please visit the journal (http://dx.doi.org/ 10.1136/bmjopen-2014006514).

Received 1 September 2014 Revised 21 October 2014 Accepted 28 October 2014

CrossMark

Faculty of Medicine \& Biomedical Sciences, School of Population Health, The University of Queensland, Herston, Queensland, Australia

Correspondence to Dr Jo Durham; m.durham@uq.edu.au

\section{ABSTRACT}

Introduction: People are increasingly mobile for numerous reasons, including healthcare. Patient mobility has vast implications for individuals, communities and whole populations and yet, to date, research on patient mobility has been quite limited. Only a small body of evidence exists on patient mobility between low-income and middle-income countries, instead having focused primarily on cross-border movement between high-income and low-income countries. In this paper, we present a protocol for examining this under-studied phenomenon.

Methods and analysis: We propose to examine patient mobility between low-income and middleincome countries using a realist synthesis approach. Specifically, we aim to document why patients from low-income and middle-income countries cross international borders for healthcare, by identifying the mechanisms through which patients decide to cross-borders, and the contextual characteristics of domestic health markets that influence this choice. An underlying theory was established, based on the lead author's experience and a brief literature review, which will provide the basis to analyse search results in a subsequent paper. Search results will be obtained from databases (Ovid Medline, EMBASE, Scopus, EconLit, Web of Science) and the grey literature. An expert committee will be enlisted, prior to screening results, to review search results to ensure comprehensiveness. Based on this preliminary theory, we propose that, in some low-income and middle-income country markets, the interaction between demand-side and supply-side determinants results in market imperfections that, in turn, lead to patient movement across borders.

Ethics and dissemination: The study does not involve primary research and, therefore, does not require formal ethical approval; we do, however, follow the relevant standards of utility, usefulness, feasibility, propriety, accuracy and accountability. The standards of realist and meta-narrative evidence synthesis (RAMESES) will be adhered to in reporting the findings of the review. Once completed, the findings of the resulting manuscript will be published in a peer-reviewed journal.

\section{Strengths and limitations of this study}

- The use of realist synthesis, which is specifically designed for use in complex systems such as health markets, is ideal for investigating questions requiring depth of understanding.

- Inclusion of a review group consisting of sector experts and researchers will help ensure complete coverage of the literature.

- The reproducibility of the review is limited due to the realist synthesis approach adopted.

Trial registration number: This protocol has been registered with PROSPERO, registration number CRD42014014391.

\section{INTRODUCTION}

Globalisation and the commodification of health are contributing to increased patient mobility as patients cross borders to access health services in neighbouring jurisdictions. Within the last decade, this international trade in cross-border health goods services has rapidly expanded. ${ }^{1}{ }^{2}$ Thus, modern health systems are a rich and complex network of interactions that cross national boundaries. ${ }^{1-3}$ The WHO inclusively defines health systems as "all the activities whose primary purpose is to promote, restore or maintain health," recognising that the health system extends beyond that of the public health sector. ${ }^{4}$ Despite this inclusive definition, most analyses are limited to an examination of the public sector within geopolitical territorial boundaries. ${ }^{3}$ Such an analysis, while attractive in terms of planning national healthcare services, distorts our understanding of the health system, which increasingly does not correspond to the notion of the health system contained within the 'nationstate'. This goes to the core of the issue that this research addresses. 
Most of the research to date has focused on 'medical tourism', which has connotations of a degree of affluence, holidays and healthcare that may not reflect the circumstances of all mobile patients. ${ }^{5}{ }^{6}$ Given this terminology, it is not surprising that research has most often documented patients in higher income countries (HICs) moving to countries with lower healthcare costs. $^{7} 8$ Patient mobility for planned healthcare is, however, also a substantial feature in low-income and middle-income countries (LMICs), whereby patients from LMICs cross international borders to HICs to purchase health services and products. ${ }^{9}$ Such cross-border health-seeking behaviour hides the true burden of disease, and impacts on domestic health markets, regulation, resource allocation and equity of access. Yet patterns of patient mobility from LMICs to HICs are poorly understood and under-researched. Further, the research that is available provides limited guidance for decisionmakers on how to adapt health policies to their objectives, target populations and local contexts, and promote equitable access.

In this research, we propose to begin to address this gap through a rapid realist synthesis of the literature. The questions we seek to answer are: Why do patients from LMICs cross international borders for healthcare? What are the contextual factors that influence this choice? Such questions are very pertinent for health systems in developing countries, and underscore the need to better understand how people behave within market systems. ${ }^{10-12}$

In this rapid review, our outcome of interest is the decision by patients to cross borders. Although important, it is beyond the scope of this review to examine the impact of these decisions on patients themselves, health services, health financing and health equity outcomes. A number of insights relating to provision of healthcare may, however, be gleaned from the current review to be further built on in future research. A realist synthesis has been selected because it is specifically designed for use in complex systems, such as health markets, and is ideal for investigating questions requiring depth of understanding. ${ }^{13-15}$ The study is planned to be undertaken from November 2014 to March 2015.

\section{Realist synthesis}

Realist synthesis is a theoretically driven, qualitative approach to synthesising qualitative, quantitative and mixed-methods research evidence. ${ }^{14}{ }^{16-21}$ A realist review is theory driven. In this review, the key theories relate to the interaction between the demand and supply-side functions in a market and health systems performance. ${ }^{11}$ While most of the realist reviews to date have focused on specific interventions or cases of interventions, in contrast, our research seeks to generate, test and refine a theory on why patients cross international borders for healthcare. This approach is appropriate, as it provides a framework to (1) examine the circumstances in which patients cross international borders and
(2) provide policy makers with information on which they can act. While systematic reviews provide evidence on outcomes and a typology of patient movements, a realist review provides a method to understand which patients decide to cross borders, how those decisions are made, and what contextual factors affect those decisions. In realist terms, these are referred to as context, mechanisms and outcomes (C-M-O configuration).

Mechanisms refer to the variables in the decisionmaking process and, more specifically, to the resources offered by the health market and the reasoning of patients who choose to seek healthcare outside the domestic market. ${ }^{15}$ They include the beliefs, values, desires and cognitive processes that influence behavioural choices. ${ }^{1416}$ These mechanisms are influenced by the context. Context, in a realist review, generally refers to aspects of the background (in this case, it is most likely the health system or health market), people and research setting that generate outcomes. ${ }^{13}{ }^{15}$ It is similar to structure, in that it is social, cultural, historical or institutional. Context allows or, conversely, constrains agency. ${ }^{22}$ Outcomes refer to expected or unexpected intermediate (mediating) and final outcomes. ${ }^{15}$ They are the result of the interaction of mechanisms and context. The C-M-O configurations help ensure external validity, as they allow the research to extend to a level of abstraction for the theory/theories to be useful in other contexts. ${ }^{1} 131722$ The iterative approach to theory building and C-M-O configuring will enable us to confirm or refute our novice theories.

\section{Research aim and objectives}

The primary aim of this synthesis is to explain why patients from LMICs cross international borders for healthcare by finding underlying theories that explain this movement. Our objectives are to

1. Document why patients from LMICs cross international borders for healthcare;

2. Identify the mechanisms generating patient decisions to cross borders;

3. Investigate the contextual characteristics of domestic health markets (demand-side and supply-side factors) that influence this choice;

4. Develop an explanatory framework that synthesises review findings of why patients cross borders.

We will address these objectives by searching for studies of cross-border patient movement to identify C-M-O configurations across studies, to produce a middle-range theory explaining why, how, for what purpose and in what circumstances patients choose to seek healthcare outside of their domestic health system. For the purpose of this review, we conceptualise health systems as market systems.

Market systems refer to the multiplayer, multifunction social arrangement consisting of three main sets of functions: demand and supply; rules or regulation; and supporting functions (eg, product development, skills improvement, research and development, coordination 
and advocacy) that are undertaken by different players, for example, private sector, government, representative organisations, civil society and so forth. Within this forum, system exchange takes place, develops, adapts and grows. ${ }^{10} 1123$ Our primary focus is healthcare services or healthcare markets.

Healthcare markets are a set of socioeconomic arrangements by which users and providers of health services are in contact to exchange goods or services, usually through some kind of formal or informal financial exchange through the process of demand and supply. ${ }^{24}$ While basic services, such as health (which may be considered a public good) may seem to be different institutions from markets, with one being seen as primarily a public service and the other being prominently private, in reality they share many of the same characteristics. The drawing of fixed lines between sectors may, therefore, be artificial, especially given most health markets are pluralistic with a mix of public and private providers. ${ }^{11} 2425$

In this review, our basic premise is that when domestic healthcare markets are imperfect, some patients seek healthcare in international markets. Our contention is that health markets in LMICs are unable to scale up the supply of healthcare goods and services to meet demand; that they are unable to meet the demand for a range of healthcare goods and services patients seek, for example, non-communicable diseases; and that the prices for healthcare goods and services are unpredictable. ${ }^{10}$ The range of factors that characterise health market failure, and the underlying mechanisms and circumstances in which patients seek healthcare in alternative international markets, will be identified. Study outcomes will be used to identify strategies for interventions to increase utilisation of domestic health markets.

\section{METHODS AND ANALYSIS}

A rapid realist review, as proposed by Saul $e t a l,{ }^{26}$ will be undertaken. The study will be conducted in the following iterative steps. We will begin by describing the initial hypotheses, or relevant candidate theories, before undertaking a more thorough search of the literature for pertinent papers, and then extracting and synthesising the data based on our theoretical framework.

\section{Identifying candidate theories}

To identify initial candidate theories, we have undertaken a preliminary exploration of the theory of how healthcare markets work, and what contributes to healthcare market failure in LMICs. The scoping review revealed a number of key elements on which a domestic healthcare market may thrive or falter. If these elements, whether stand-alone, or in conjunction, are (or perceived to be) insufficient, patients may seek alternative services, which could include going abroad.

A healthcare market is an interactive socioeconomic institution that works through the process of interactions of demand and supply. ${ }^{10232427} 28$ Healthcare markets should work by stimulating choice and competition so that providers continually work to improve their efficiency and products, and meet consumer demand. ${ }^{102328}$ When operating under conditions of perfect competition and perfect information, rational market players respond to price signals, and this price-regulated, money-based exchange ensures efficiency and equilibrium between supply and demand. ${ }^{10} 1129$ Markets, however, often work imperfectly, leading to market failure. ${ }^{10} 1129$ What constitutes 'health system failure' is not, however, clear cut. ${ }^{30}$ Despite this, we have identified in the literature a number of health system features that may have adverse implications for health, including six health systems performance themes, namely accessibility and responsiveness; quality; outcomes; accountability, transparency and regulation; fairness and equity; and efficiency, identified by the WHO. ${ }^{25}$

Demand for healthcare is dictated by the demand for health, which is driven by a number of factors, including demographic, lifestyle and health. ${ }^{31}$ Demand-side factors are those factors that influence demand and operate at the individual, household or community level. ${ }^{32}$ One demand-side barrier is the affordability, or cost, of healthcare services and products. ${ }^{33-36}$ Costs include formal and informal charges, as well as opportunity costs. ${ }^{35}$ Other demand-side barriers relate to accessibility, including distance and access to transport, and adequacy and acceptability, in terms of perceptions of quality of care. ${ }^{36}$ Another demand-side determinant relates to consumer satisfaction. This refers to consumer attitudes towards healthcare services, based on past contact with healthcare services. ${ }^{33} 34$ It includes perceived convenience of care, coordination and cost, courtesy of health staff, information given to the patient about dealing with illness, and subjective assessment of the quality of the care received. ${ }^{33}$ Demand is also influenced by perceptions of illness and need, and willingness to seek services. ${ }^{32}$ Availability can also act as a demand-side barrier, particularly in LMICs, where a patient's choice concerning the range of healthcare goods and services is limited by supply creating demand, rather than vice versa. Availability, however, may also be a supply-side determinant. ${ }^{10} 36$

Supply-side determinants of healthcare services are those that arise from the healthcare production function, and that interact to produce effective healthcare services. ${ }^{10} 32$ Supply-side factors relate to (1) the quality of staff, and include whether they speak the same language as the target population, their availability to provide services, and their ability to treat certain conditions; (2) the quality and availability of buildings, technology and equipment; (3) the price, availability and quality of consumables and (4) the quality of management. ${ }^{32}$ Together, demand-side and supply-side factors result in a demand for healthcare of a given quality that is determined by individual and community factors, and the cost of healthcare and related goods. ${ }^{10} 2332$ 


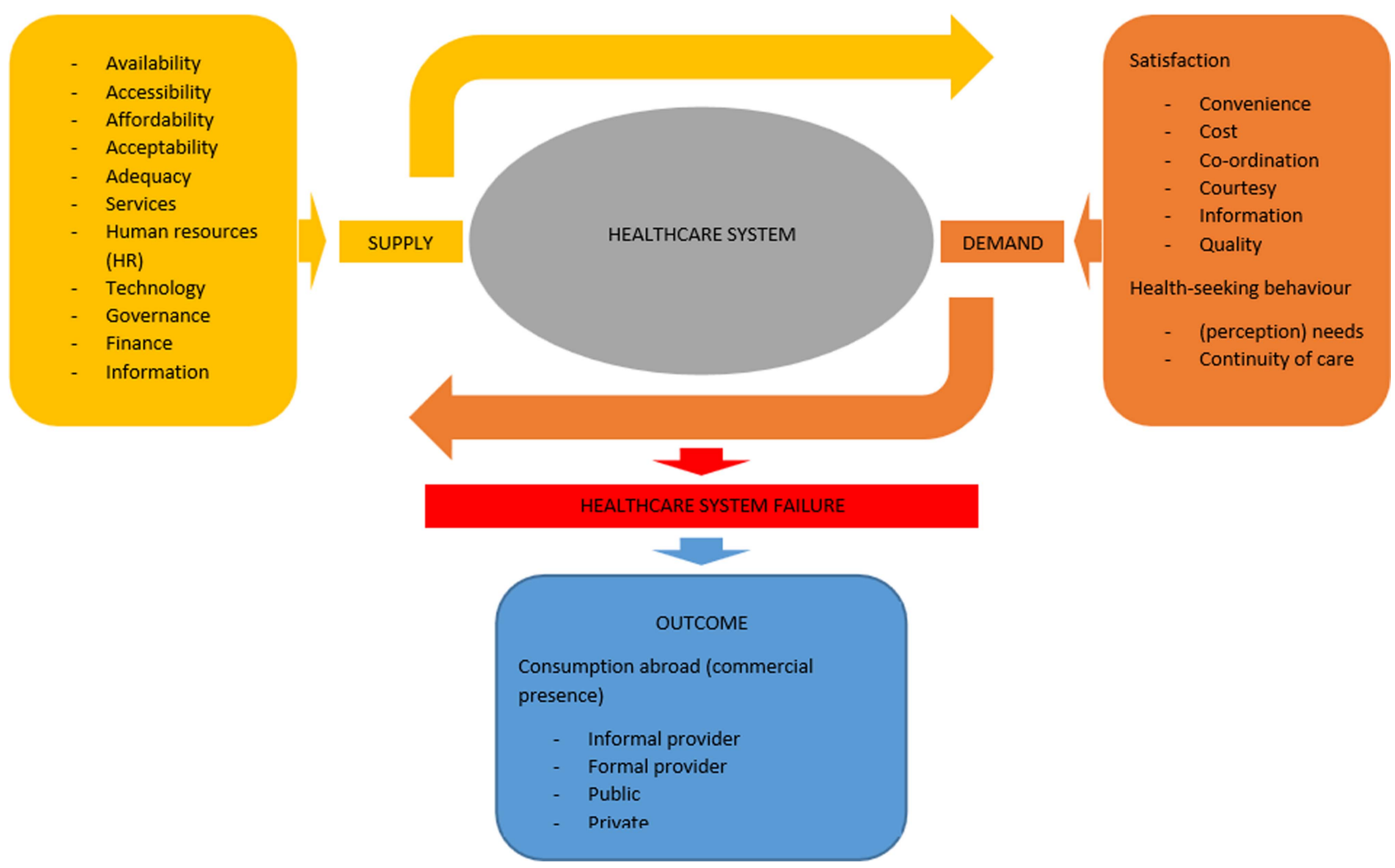

Figure 1 Preliminary theory.

Based on the above, we propose that, in some LMIC markets, the interaction between demand-side and supply-side determinants results in market imperfections that, in turn, lead to patient movement across borders. This working theory is illustrated in figure 1 .

The theory will then be examined using a realist mode of analysis involving the concepts of C-M-O configuration. The outcome of interest is utilisation of healthcare services across geopolitical state borders. Based on this preliminary scoping of the literature, we aim to further elucidate: (1) a theory of domestic health system failure and its relationship to patient movement across borders; (2) the identification of the contextual factors that influence patients to cross international borders and (3) the effects of patient movement across borders. This will be done in an exploratory, non-exhaustive and inductive manner. Through this process, we will develop a framework based on the C-M-O principle.

\section{Realist review}

Following Saul $e t a l,{ }^{26}$ a review group consisting of sector experts and researchers is currently being established. Identification of articles and documents for inclusion in the review will include generating a list of relevant documents with the review group. The next step will be to undertake a rapid realist review of the literature to further map the elements of, and approaches to, health markets and trans-border patient movement, with a view to further uncovering the underlying theory. This search will be undertaken using databases such as the Ovid Medline, EMBASE, Scopus, EconLit, Web of Science and web-based searches of data available in the public domain. Combinations of key words in English, and their truncations, will be entered in these databases; see online supplementary file 1 for preliminary search terms. The search terms will be iteratively narrowed based on the results that are most relevant to the current review. ${ }^{26}$

The relevance of the retrieved documents will be assessed according to exclusion and inclusion criteria, and how each study clarifies the C-M-O configurations. Bibliographic references from the included documents will be reviewed, using the snowballing technique to identify additional documents as well as reference and citation tracking. ${ }^{13}{ }^{37}$ We will continue to identify potential documents through this procedure throughout the review process. Only studies written in English will be included due to budgetary constraints. The search will be further limited to studies published between 2000 and 2014. The search for new documents will end at the point of theoretical saturation-in other words, when the research yields no more sources producing new aspects of the theory. Working with the review group will help ensure that we do not omit significant sections of the literature. ${ }^{26}$ References will be compiled in EndNote. To aid in sifting documents, we will be guided by the following questions:

1. Does the study describe a health services market? 
2. Is the study implicitly or explicitly underpinned by the theory of health services markets and demand and supply functions?

3. Does the study provide information on demand-side and/or supply-side context of the domestic market?

4. Does the study provide evidence that will contribute to the synthesis and our emerging theory?

Following the RAMESES (realist and meta-narrative evidence synthesis) guidelines for realist synthesis, quality assessment will be based on how each study clarifies the C-M-O configurations. ${ }^{38}$ In realist synthesis, the criteria for quality assessment are relevance and rigour. ${ }^{14}$ Relevance refers to relevance to a specific aspect of the theory under test. Rigour relates to methodological rigour in relation to the specific finding or inference which is drawn on in the review (as opposed to the overall rigour of the whole study). ${ }^{13}$ The Mixed Methods Appraisal Tool ${ }^{39}$ will be used to assist in the assessment of the quality of studies, but with the criteria applied to the relevant aspects of the studies, rather than the studies as a whole. ${ }^{13}$ To ensure a transparent process, we will develop a summary table specifying the authors, objectives, type of study, different methodological aspects and study country. ${ }^{13}$

\section{Data extraction}

The documents included in the review will be compiled in Excel. The variables extracted will be (1) author and year of publication; (2) patient sending and receiving countries; (3) the socioeconomic and political contextual variables influencing decisions to seek healthcare abroad (at country, health system and individual level); (4) beliefs, values, desires and cognitive processes influencing behaviour (mechanisms) and (5) outcomes.

\section{Data synthesis}

The analytical framework developed in the first step will be used to analyse the data, and will be further specified in an iterative manner, in order to integrate new explanatory elements categories. Each primary study will be examined for evidence based on how it supports, refutes, reinterprets or refocuses the novice theories developed in the first step, looking at how each study contributes to refining the initial theory. In this way, using an abductive approach, that is combining methods of inductive and deductive analytical processes to developing theory, it will be possible to specify C-M-O configurations, with each study helping to clarify or reformulate the $\mathrm{C}-\mathrm{M}-\mathrm{O}$ configurations, in order to take into account potential contradictory elements. Following the RAMESES guidelines, the intent will not be to provide a quantifiable summary of the abstracted variables but rather to interrogate the interaction between context, mechanism and outcomes.

\section{Validity}

The construction of explanations to make 'sense' of patient mobility, through an iterative process between empirical data and construction of C-M-O configurations, enhances internal validity, as does the use of an appraisal tool for quality assessment. The inclusion of context in our analysis increases the generalisation potential of the study. Further, the theories generated by the review will be examined by the review group, and will help to fill any gaps in the published literature. ${ }^{26}$ An audit trail will be maintained to help ensure transparency.

\section{ETHICS}

As the study does not involve primary research, it does not require formal ethical approval. The study will, however, follow the relevant standards of utility, usefulness, feasibility, propriety, accuracy and accountability. ${ }^{40}$

\section{DISCUSSION}

\section{Significance of the proposed research}

Cross-border healthcare has implications for health equity. While there have been several studies looking at medical tourism, few studies have considered how market failure contributes to patient cross-border movement in LMICs. The results of the proposed research will be useful to health policy makers in increasing utilisation of domestic health services. Further, while realist reviews are increasing, their use by public health researchers is relatively new. ${ }^{41}$

\section{Dissemination}

The standards of RAMESES will be followed in reporting the findings of this review. ${ }^{38}$ The findings will also be published in a peer-reviewed journal that specialises in health policy, and with a particular focus on LMICs.

\section{Limitations}

As with all research methods, realist reviews have some limitations. One is that, compared to systematic reviews, realist reviews are harder to reproduce, as selecting papers and following candidate theories require judgement, often based on a mixture of experience, intuition and prudence, to identify those with greatest relevance. ${ }^{26}$ In order to ensure transparency, however, we will include a summary table and methodological details of papers included in the review. It could be argued that a limitation of a realist review is that it does not provide certitude in terms of findings. From a realist perspective, however, this is not seen as a limitation, as there is no such thing as final truth or knowledge. Rather, a realist review aims to offer insights into how decisions might be made or how interventions might fare in certain circumstances, and what the outcomes might be for whom. ${ }^{26} \mathrm{~A}$ limitation of our study is that only studies written in English will be included and this may introduce an element of language bias. The cost of translation, however, is beyond the scope of this study and the feasibility and accuracy of free web-based translation tools has not yet been determined for the purpose of realist reviews. 


\section{Conclusion}

Health systems are complex, and even more so in an increasingly globalised world. In this paper, we present a protocol for conducting a realist synthesis on patient mobility between LMICs, which has important, but under-studied, implications for healthcare systems. We have formulated an initial theory of cross-border healthcare use, based on domestic health system (perceived) inadequacy, in terms of supply-side and demand-side factors. This theory will inform an analysis of international cross-border patient mobility by patients from LMICs for healthcare, the results of which may better inform health policy makers (to be presented in a subsequent paper). Understanding 'real world' health system use is imperative for optimal outcomes, health and otherwise, for individuals, communities and populations in the country of origin and abroad.

Contributors JD conceived the original idea, formulated the preliminary theory, drafted the manuscript and approved the final document; SJB contributed to the formulation of the preliminary theory, drafted the manuscript and approved the final document.

Funding This research received no specific grant from any funding agency in the public, commercial or not-for-profit sectors.

Competing interests None.

Provenance and peer review Not commissioned; externally peer reviewed.

Data sharing statement Required data/information may be requested from the corresponding author.

Open Access This is an Open Access article distributed in accordance with the Creative Commons Attribution Non Commercial (CC BY-NC 4.0) license, which permits others to distribute, remix, adapt, build upon this work noncommercially, and license their derivative works on different terms, provided the original work is properly cited and the use is non-commercial. See: http:// creativecommons.org/licenses/by-nc/4.0/

\section{REFERENCES}

1. Whittaker A. Cross-border assisted reproduction care in Asia: implications for access, equity and regulations. Reprod Health Matters 2011;19:107-16.

2. Glinos IA, Baeten R, Helble M, et al. A typology of cross-border patient mobility. Health Place 2010;16:1145-55.

3. Zanini G, Raffaetà R, Krause K, et al. Transnational medical spaces opportunities and restrictions. MMG Working Paper 13-16. Göttingen, Germany: MMG, 2013.

4. World Health Organization. Everybody's business: strengthening health systems to improve health outcomes: WHO's framework for action. Geneva: World Health Organization, 2007 (cited 20 October 2012). http://www.who.int/healthsystems/strategy/everybodys_

5. Kangas $B$. The burden of pursuing treatment abroad: three stories of medical travellers from Yemen. Global Soc Policy 2010;10:306-14

6. Kangas B. Travelling for medical care in a global world. Med Anthropol 2010;29:344-62.

7. Whittaker A. Pleasure and pain: medical travel in Asia. Global Public Health 2008;3:271-90.

8. Lunt N, Mannion R. Patient mobility in the global marketplace: a multidisciplinary perspective. Int J Health Policy Manag 2014;2:155-7.

9. Crush J, Chikanda A. South-South medical tourism and the quest for health in Southern Africa. Soc Sci Med 2014 In press. doi:10.1016/j.socscimed.2014.06.025

10. Ghosh BN. Rich doctors and poor patients: market failure and health care systems in developing countries. J Contemp Asia 2008;38:259-76.

11. Tschumi $\mathrm{P}$, Hagan $\mathrm{H}$. A synthesis of the making markets work for the poor (M4P) approach. UK Department for International Development (DFID) and Swiss Agency for Development and Cooperation (SDC), 2008.
12. Bloom $\mathrm{G}$, Standing $\mathrm{H}$, Lucas $\mathrm{H}$, et al. Making health markets work better for poor people: the case of informal providers. Health Policy Plan 2011;26(Suppl 1):i45-52.

13. Westhorp G, Walker B, Rogers P. Under what circumstances does enhancing community accountability and empowerment improve education outcomes, particularly for the poor? A realist synthesis protocol. London: EPPI-Centre, Social Science Research Unit, Institute of Education, University of London, 2012.

14. Pawson R. Evidence-based policy: a realist perspective. London Sage, 2006

15. Wong G, Westhorp G, Pawson R, et al. Realist synthesis: Rameses training materials, 2013. http://www.ramesesproject.org/media/ Realist_reviews_training_materials.pdf

16. Best A, Greenhalgh T, Lewis S, et al. Large-system transformation in health care: a realist review. Milbank Q 2012;90:421-56.

17. Geoff W. Internet-based medical education: a realist review of what works, for whom and in what circumstances. BMC Med Educ 2010;10:12

18. Jagosh J, Pluye P, Macaulay A, et al. Assessing the outcomes of participatory research: protocol for identifying, selecting, appraising and synthesizing the literature for realist review. Implement $\mathrm{Sci}$ 2011;6:24

19. Pawson R. Evidence-based policy: the promise of "realist synthesis". Evaluation 2002;8:340-58.

20. Pawson R, Greenhalgh T, Harvey G, et al. Realist synthesis: an introduction. Manchester: ESRC Research Methods Programme, University of Manchester, 2004

21. Pawson R, Greenhalgh T, Harvey G, et al. Realist review-a new method of systematic review designed for complex policy interventions J Health Serv Res Policy 2005;10(Suppl 1):21-34.

22. Robert E, Ridde V, Marchal B, et al. Protocol: a realist review of user fee exemption policies for health services in Africa. BMJ Open 2012;2:e000706.

23. Bloom $\mathrm{G}$, Standing $\mathrm{H}$, Lucas $\mathrm{H}$, et al. Making health markets work better for poor people: the case of informal providers. Health Policy Plan 2011;26:i45-52.

24. Bloom G, Henson S, Peters DH. Innovation in regulation of rapidly changing health markets. Global Health 2014;10:53

25. Basu S, Andrews J, Kishore S, et al. Comparative performance of private and public healthcare systems in low-and middle-income countries: a systematic review. PLoS Med 2012;9:e1001244.

26. Saul J, Willis C, Bitz J, et al. A time-responsive tool for informing policy making: rapid realist review. Implement Sci 2013;8:103.

27. Bennett S, Bloom G, Knezovich J, et al. The future of health markets. Global Health 2014;10:51.

28. Davidson B. Contestability in human services markets. J Aust Polit Econ 2011;68:213-39.

29. Mwachofi A, Al-Assaf AF. Health care market deviations from the ideal market. Sultan Qaboos Univ Med J 2011;11:328-37.

30. Lutschini M. Engaging with holism in Australian Aboriginal health policy-a review. Aust New Zealand Health Policy 2005;2:15

31. Jack EP, Powers TL. A review and synthesis of demand management, capacity management and performance in health-care services. Int J Manag Rev 2009;11:149-74.

32. Ensor T, Cooper S. Overcoming barriers to health service access: influencing the demand side. Health Policy Plan 2004;19: 69-79.

33. Aday LA, Andersen R. A framework for the study of access to medical care. Health Serv Res 1974;9:208-20.

34. Andersen R, McCutcheon A, Aday LA, et al. Exploring dimensions of access to medical care. Health Serv Res 1983;18:49-74.

35. Ensor T, Cooper S. Overcoming barriers to health service access and influencing the demand side through purchasing. Heslington, York: World Bank, 2004.

36. Obrist B, Iteba N, Lengeler C, et al. Access to health care in contexts of livelihood insecurity: a framework for analysis and action. PLoS Med 2007; 4:e308.

37. Greenhalgh T, Peacock R. Effectiveness and efficiency of search methods in systematic reviews of complex evidence: audit of primary sources. BMJ 2005;331:1064-5.

38. Wong G, Greenhalgh T, Westhorp G, et al. RAMESES publication standards: realist syntheses. BMC Med. 2013;11:21.

39. Pluye P, Robert E, Cargo M, et al. Proposal: a mixed methods appraisal tool for systematic mixed studies reviews 2011 [cited 24 August 2014]. http://mixedmethodsappraisaltoolpublic.pbworks.com

40. Mertens DM. Transformative research and evaluation. New York: The Guilford Press, 2010.

41. Marchal B, Dedzo M, Kegels G. A realist evaluation of the management of a well-performing regional hospital in Ghana BMC Health Serv Res 2010;10:24. 